\title{
Demodulation of aggregated RF Signals with a Unique Rx Chain
}

\author{
A. Kaissoine, B. Huyart and A. Mbaye \\ Communication \& Electronique Dpt \\ Institut Télécom, Télécom-ParisTech, \\ Paris-France \\ abdou.kaissoine@ telecom-paristech.fr \\ huyart@telecom-paristech.fr
}

\author{
K. Mabrouk \\ AllianSTIC \\ ESIGETEL \\ Fontainebleau-France \\ kais.mabrouk@esigetel.fr
}

\begin{abstract}
In the next generation of the mobile radio systems, the information will be distributed on discontinuous frequency bands. To demodulate the RF signal, a possible architecture is to use a number of receivers $R x$ equal to that of the discontinuous RF frequency bands. This paper demonstrates that this operation can be made with a single receiver $R x$. The method is based on the mixing of the RF signal with a local oscillator (LO) signal constituted by several Continuous Wave (CW) signals. The technique is studied theoretically and then applied to the case of RF signal constituted of two frequency bands. The $\mathrm{CW}$ frequencies of the LO signal are selected to convert both bands of the RF signal in Zero Intermediate Frequency (ZIF) and LOW Intermediate Frequency (LIF).
\end{abstract}

\section{INTRODUCTION}

The International Telecommunication Union of Radiocommunication (ITU-R) recommended that the 4th generation network must reach the data rate of $1 \mathrm{Gbit} / \mathrm{s}$ for the downlink (base station to mobile station) and $500 \mathrm{Mbit} / \mathrm{s}$ for the uplink (mobile station to base station)[1],[2]. This data rate requires a frequency bandwidth between $70 \mathrm{MHz}$ and 100 $\mathrm{MHz}$. The availability of this frequency range is not always guaranteed, especially for RF carrier frequency below $3 \mathrm{GHz}$. The solution is spectrums aggregation which consists in distributing the information on multiple discontinuous frequency bands. Nowadays, the proposed architecture is to use a number of Rx receiver equal to the number of frequency bands of the RF signal. In the case of 2 bands, another way is to use the architecture of the Weaver demodulator and a LO frequency equals approximately to the half of both carrier frequencies [3], [4]. This last technique is not applicable for the demodulation of more than 2 frequency bands.

In parallel with our research works, a team of NTT published in [5] practical results on the transposition by a mixer of a RF signal constituted of 2 frequency bands. The used technique is similar to that developed in this paper and consists in using a LO signal constituted of 2 frequency tones. It is shown in [5] that power levels of the IF signals may be identical even if the RF signal is composed of two frequency bands of different power levels. This property is obtained by monitoring the relative power of $\mathrm{CW}$ signals of the LO. However, that involves a degradation of the signal to noise ratio for one of the bands by increasing the conversion loss of the mixer.

In the work presented in this paper this technique is applied directly for the demodulation of RF signal aggregated in frequency and without any control of the power level of the LO signal.

The principle of transposition in baseband of a RF signal constituted of discontinuous frequency bands is demonstrated in section II. Finally the section III gives a validation of the theoretical results by the measure. The variation of the quality of the In phase and Quadrature (IQ) constellation of the demodulated signals is given according to the relative power level of both frequency bands of the RF signal.

\section{TheORY AND TECHNiQue Of The Down CONVERSION OF THE RF SIGNAL}

The transposition of a RF signal to baseband is done by the mixing with a LO signal. Assuming the RF signals is constituted of 2 discontinuous frequency bands, respectively centered at the frequencies $f_{l}$ and $f_{2}$ and of bandwidth $B W_{l}$ and $B W_{2}$; the expression of the aggregated RF signal is expressed thus:

$$
v_{R F}(t)=\operatorname{Re}\left[Z_{1}(t) e^{j 2 \pi f_{1} t}+Z_{2}(t) e^{j 2 \pi f_{2} t}\right]
$$

with $Z_{j}(t)=a_{j}(t) e^{j \theta_{j}(t)}, j=1,2$ the complex envelope of 2 carrier frequencies $f_{1}$ and $f_{2}$.

In our case the objective is to lower the frequency bandwidth of the converted signals to relax the required performances of the Analog to Digital Converter (ADC). The strategy is consequently to convert both frequency bands of the RF signal to ZIF for one band and LIF at frequency $\Delta f_{2}$ for the second one. In that way, both $\mathrm{CW}$ frequencies of the LO 
signal are equal to $f_{1}$ and $f_{2^{-}} \Delta f_{2}$ with $\Delta f_{2}>B W_{1} / 2$. The expression of the LO signal is defined as follows:

$$
v_{L O}(t)=\operatorname{Re}\left[V_{L O}\left(e^{j\left(2 \pi f_{1} t+\phi_{1}\right)}+e^{j\left(2 \pi\left(f_{2}-\Delta f_{2}\right) t+\phi_{2}\right)}\right)\right]
$$

with $V_{L O}$ the amplitude of the $\mathrm{CW}$ signals and angles $\phi_{i}$ $(i=1,2)$ representing phase shifts between RF and LO signals. Assuming that $f_{2}>f_{1}$ so that we obtain the following condition:

$$
\left|f_{2}-f_{1}\right|>f_{L P F}
$$

where

$$
f_{L P F}=\Delta f_{2}+B W_{2} / 2
$$

is the cutoff frequency of the low-pass filter.

The expression to the output signal of the low-pass filter is expressed as follows:

$$
v_{k}(t)=V_{L O}\left\{\begin{array}{l}
A_{k 1} a_{1} \cos \left(\theta_{1}(t)-\phi_{1}\right)+ \\
A_{k 2} a_{2} \cos \left(\theta_{2}(t)+2 \pi \Delta f_{2} t-\phi_{2}\right)
\end{array}\right\}
$$

where $A_{k j}, j=1,2$ are gains that include the conversion gain of the mixer and the losses of splitters, phase shifters and filters. And $\mathrm{k}$ represents the output path of the mixer.

The equation (5) shows that the first frequency band is transposed directly to baseband around zero, it is a ZIF demodulation. The second band is transposed to the intermediate frequency $\Delta f_{2}$, it is a LIF demodulation.

Moreover the separation between both baseband spectrums is possible if:

$$
\Delta f_{2}-B W_{2} / 2>B W_{1} / 2
$$

This technique is applied to the Three Phase Demodulator (TPD) instead of the Classical IQ (CIQ) demodulator. Indeed, a previous paper [6] has shown TPD gives a gain of $20 \mathrm{~dB}$ in second-order inter-modulation distortion (IMD2) rejection in comparison with CIQ. The operation mode of TPD differs from the CIQ. The RF and LO signals are divided into three paths instead of two. The phase shift between the LO signals is equal to $\pm 120^{\circ}$ for the TPD instead of $90^{\circ}$ for the CIQ. The CIQ delivers directly the IQ baseband signals. In the case of the TPD, the output signals are three low frequency signals, named $v_{l}(t), v_{2}(t)$ and $v_{3}(t)$. The TPD (Fig. 1) was essentially realized using COTS components. The phase shifters were made with microstrip propagation lines. The phase shift varies from $60^{\circ}$ to $150^{\circ}$ in function of frequency in the range 2 to 4.2 GHz. The splitters insertion loss is $5 \mathrm{~dB}$. The mixers present the following characteristics: RF/LO inputs ( $\mathrm{DC}=2-7$ $\mathrm{GHz}$, FI output (DC-1.3 GHz), LO power $(7 \mathrm{dBm})$, Conversion Loss $(7 \mathrm{~dB})$ and intercept point of order 2 (IIP2 = $27 \mathrm{dBm})$.

[7], [8] demonstrate that if $v_{2}(t)$ output is symmetric in comparison with 2 others, the three output signals of the TPD can be added by an analog circuit of IQ regeneration performing the following operations:

$$
\begin{gathered}
I_{\text {out }}(t)=\mu_{I} \cdot\left[-V_{1}(t)+2 \cdot V_{2}(t)-V_{3}(t)\right] \\
Q_{\text {out }}(t)=\mu_{Q} \cdot\left[V_{1}(t)-V_{3}(t)\right]
\end{gathered}
$$

Both constants $\mu_{I}$ and $\mu_{Q}$ are determined by a classical equalization procedure. In this work this operation is done thanks to a training sequence. Finally the transmitted $I(t)$ and $Q(t)$ signals are recovered.

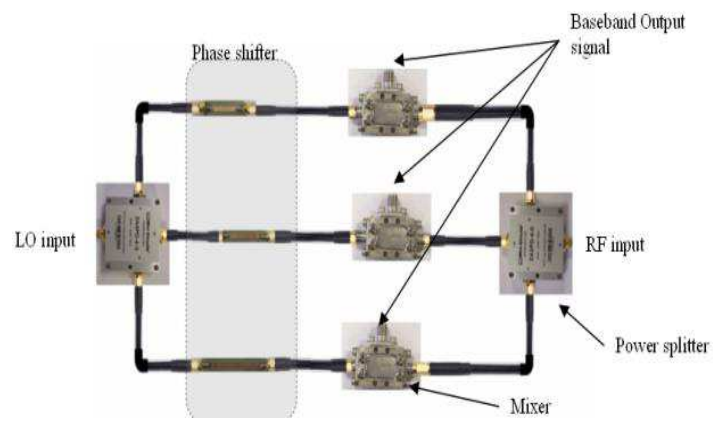

Fig. 1. Three Phase Demodulator

The mismatch between the paths of the demodulator depends upon the deviation in symmetric conditions of amplitude and phase. In [7], the simulated results demonstrate that a phase asymmetry at port 3 of value $\Delta \Phi$ introduces an amplitude error on IQ output. For example, a phase imbalance, $\Delta \Phi= \pm 10^{\circ}$ gives $\pm 3 \mathrm{~dB}$ imbalance IQ output as shown in the Fig.3 of [7].

\section{MEASUREMENT RESUlTS}

The Fig. 2 presents the test bench. The digital $I(p)$ and $Q(p)$ data are generated from Matlab software. These data are transmitted to both generators (Agilent Technologies E8267) via a Local Area Network (HP J2600A HUB). The generators operate in the mode ARB (arbitrary waveform generator). The modulated signals are added by a combiner (PULSAR PS-20$450 / 10 S$ ). In our experience, the RF signal is the aggregation of two modulated signals of carrier frequencies $2 \mathrm{GHz}$ and 2.3 GHz. The numerical frame is composed of a 16 symbols training sequence with Constant Amplitude Zero Autocorrelation (CAZAC) followed by a pseudorandom PN9 data sequence. The modulation format is Quadrature Phase Shift Keying (QPSK) with a symbol rate of $100 \mathrm{kS} / \mathrm{s}$ for each aggregated signal. The $I(p)$ and $Q(p)$ data are filtered by a Square Root Raised Cosine filter (SQRC) with a roll-off equal to 0.35 .

The test is performed using baseband signals with low frequency bandwidths. However, the TPD can also demodulate RF signals with modulation signals of high bandwidth. 


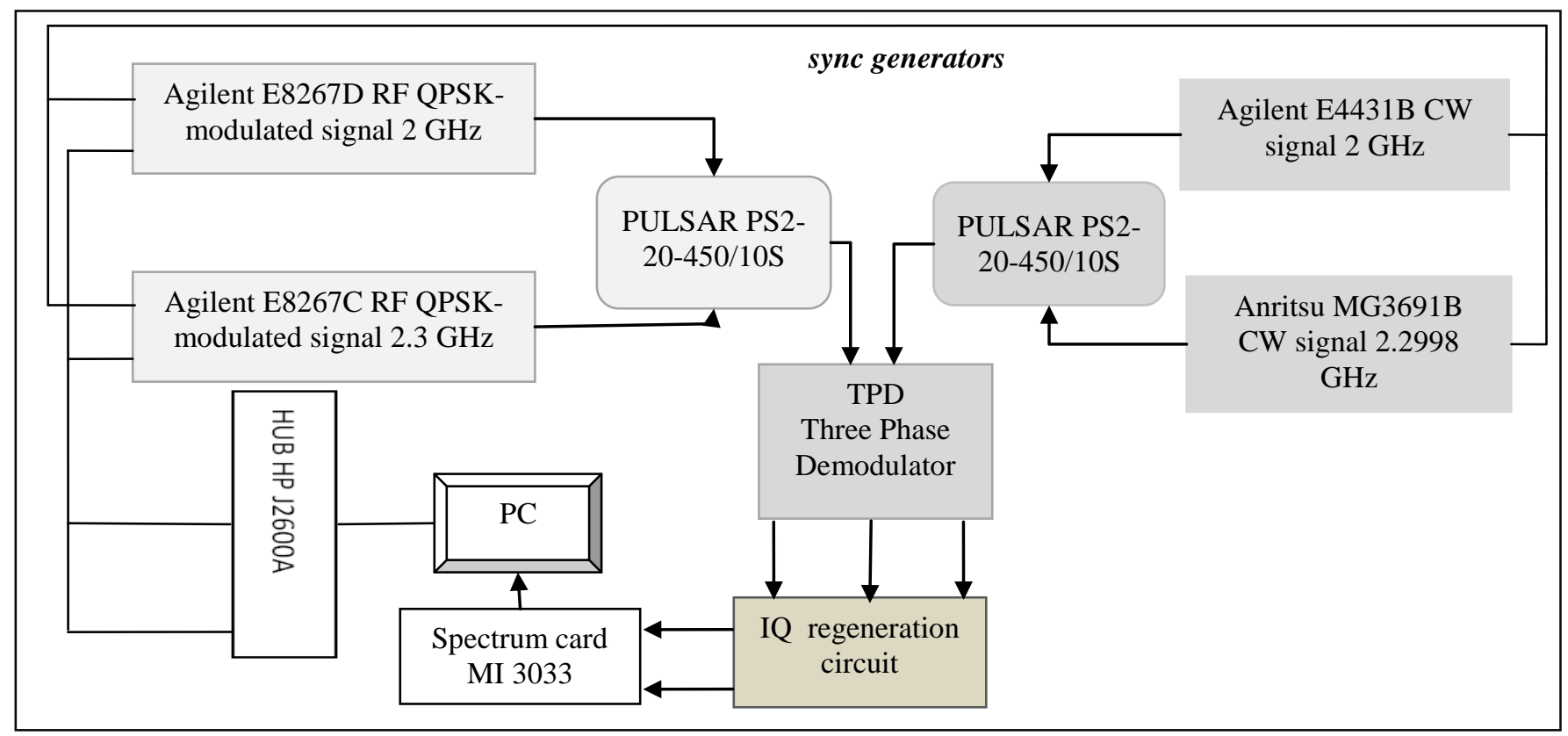

Fig. 2. Test Bench for the demodulation of RF signal aggregated in frequency

For example, Fig. 3 shows the measured baseband spectrums of a RF signal constituted of carrier frequencies 2 and $2.3 \mathrm{GHz}$ modulated with signals of $20 \mathrm{MHz}$ bandwidth. The digital signals are generated and filtered by a SQRC of roll-off equal to 0.35 . The latter reduces the frequency band of the RF signal. Thus, the spectrum of the output signal $V_{3}$ of the TPD is composed of two bands: $0-13.5 \mathrm{MHz}$ (ZIF configuration) and $36.5-63.5 \mathrm{MHz}$ (LIF configuration) (Fig. $3)$.

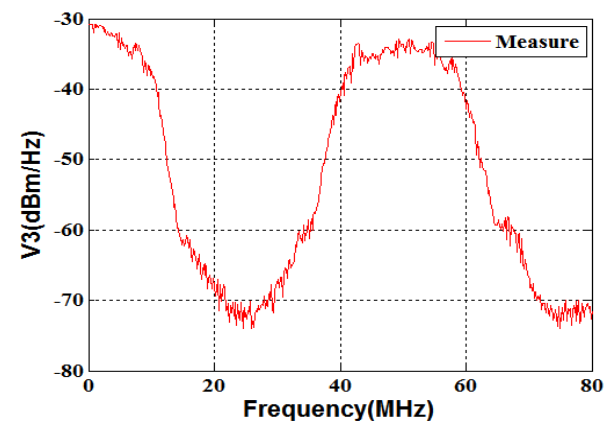

Fig. 3. Power Spectral Density in $\mathrm{dBm} / \mathrm{Hz}$ of $\mathrm{V}_{3}$ signal in function of frequency for bandwidth to $20 \mathrm{MHz}$.

Due to components limitations (data acquisition card, Low Frequency amplifiers, PC...) the measurements were made with data rate of $100 \mathrm{kS} / \mathrm{s}$.

The frequency conversion of the aggregated RF signal is performed by the mixing with a LO signal constituted of two $\mathrm{CW}$ signals. According to section II, the first $\mathrm{CW}$ frequency is $2 \mathrm{GHz}$ to convert the first band to ZIF and the second $\mathrm{CW}$ frequency is $2.2998 \mathrm{GHz}$ to convert the second band to LIF. The synchronization of the generators is performed by a common clock signal of frequency $10 \mathrm{MHz}$ (Fig. 2).
The RF signal is demodulated by the TPD. The three outputs signals of the TPD are combined in the IQregeneration circuit (Fig. 2).

The spectrum of the baseband Iout signal shows two distincts bands centered respectively between $0-67 \mathrm{kHz}$ (ZIF configuration) and 133-267 kHz (LIF configuration) (Fig. 4).

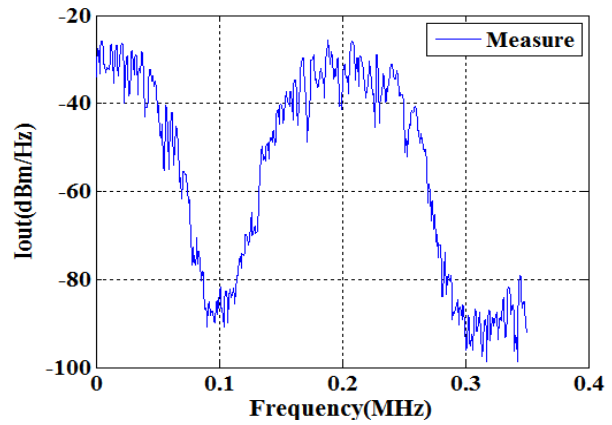

Fig. 4. Power Spectral Density in $\mathrm{dBm} / \mathrm{Hz}$ of Iout signal in function of frequency for bandwidth to $100 \mathrm{KHz}$.

The sampling frequency of the data acquisition card (MI3033) is equal to $800 \mathrm{KHz}$. The Iout and Qout signals are oversampled with a factor equal to 8 . Then equalization of $\mathrm{I}_{\text {out }}$ and $\mathrm{Q}_{\text {out }}$ is performed using the training sequence. Fig. 5 shows that the two QPSK constellations are recovered and centred. The red points show that the synchronization with the CAZAC sequence functions correctly. These results validate the theoretical assumptions developed in II.

The performances of this receiver are evaluated using the Error Vector Magnitude (EVM). We call ZIF signal (resp. LIF), the signal of carrier frequency $2 \mathrm{GHz}$ (resp. $2.3 \mathrm{GHz}$ ), which is transposed to ZIF (resp. LIF). The results show a gain of 2 to $3 \mathrm{~dB}$ in sensitivity (defined here for EVM=$15 \mathrm{~dB}$ ), for the LIF signal in comparison with the ZIF signal (Fig. 6). 

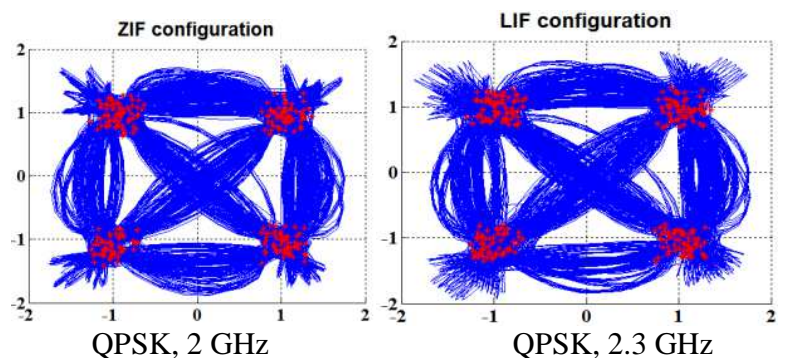

Fig. 5. Phase constellation of 2 discontinuous frequency bands RF signal translated Zero-IF and $200 \mathrm{KHz}$ LIF

The results of the EVM presented in the Fig. 6 were performed by varying simultaneously the input power level of the ZIF and LIF signals. The Fig. 7 and the Fig. 8 show the scenarios where one of the power level of a signal (ZIF or $\mathrm{LIF}$ ) is fixed to $-47 \mathrm{dBm}$ and the other varies.

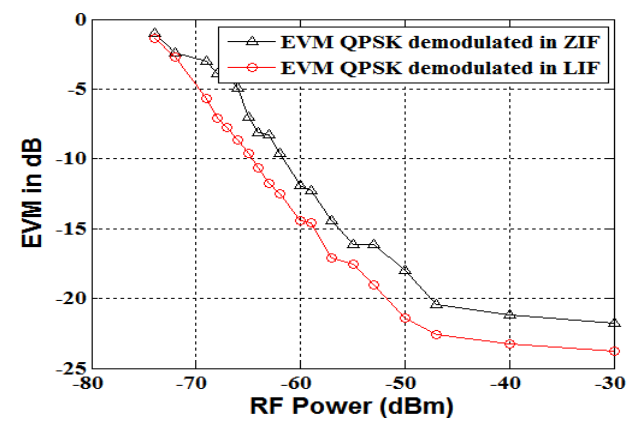

Fig. 6. EVM in $\mathrm{dB}$ in function of RF input signal in $\mathrm{dBm}$ constituted of 2 carriers modulated by QPSK signals.

By comparing the EVM results of ZIF signal in Fig. 6 and Fig. 7, there is a degradation of about $5 \mathrm{~dB}$ when the fixed ($47 \mathrm{dBm}$ ) power level of the LIF signal is greater than the power level of the ZIF signal. For the same limit value of -47 $\mathrm{dBm}$, there is only a drop of about $2.5 \mathrm{~dB}$ for the EVM of the LIF signal in Fig. 6 and Fig. 8. Thus the LIF signal influences more the quality of the converted ZIF signal. This can be explained by second-order inter-modulation distortion (IMD2), which is more important in the ZIF converted band than in the LIF one. This effect has been checked by theoretical study and by spectrum measurement but is not described here due to space limit of the paper.

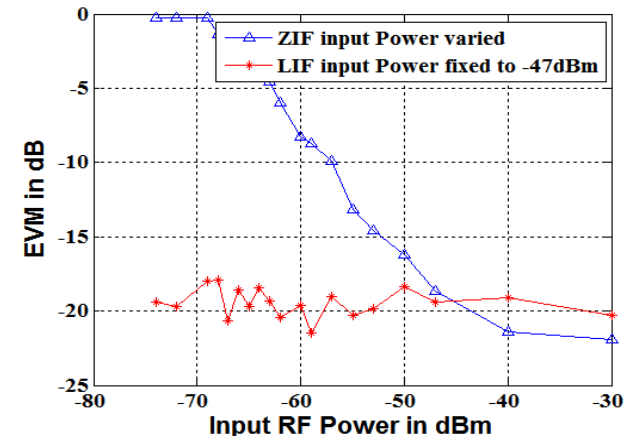

Fig. 7. EVM in $\mathrm{dB}$ in function of RF input signal in $\mathrm{dBm}$ constituted of 2 carriers modulated by QPSK signals, so the power of signal RF to frequency $2.3 \mathrm{GHz}$ fixed to $-47 \mathrm{dBm}$.

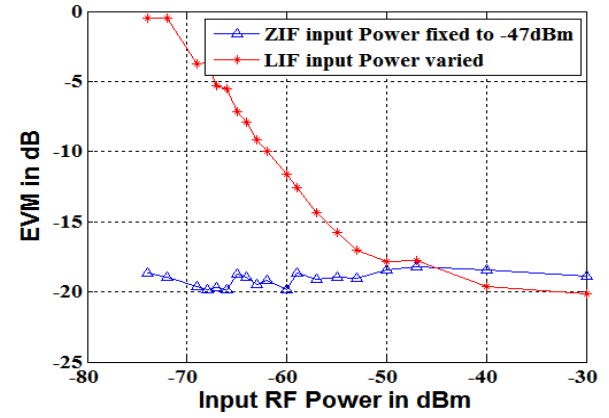

Fig. 8. EVM in $\mathrm{dB}$ in function of RF input signal in $\mathrm{dBm}$ constituted of 2 carriers modulated by QPSK signals, so power of signal RF to frequency 2 $\mathrm{GHz}$ fixed to $-47 \mathrm{dBm}$.

\section{CONCLUSION}

This paper demonstrates that it is possible to demodulate a RF signal constituted of two discontinuous frequency bands with a single Rx chain. However, this technique can be extended to a greater number of bands. The used technique is based on a LO signal composed of several CW signals. The used method is demonstrated theoretically and confirmed by measurements results. The demonstration has been done for two useful signals centered at $2 \mathrm{GHz}$ and $2.3 \mathrm{GHz}$ and QPSK modulated with a symbol rate of $100 \mathrm{Ks} / \mathrm{s}$. The signals have been respectively converted to ZIF and LIF in such a way the $\mathrm{ADC}$ has to convert low frequencies signals of frequency bandwidth $300 \mathrm{kHz}$.

\section{REFERENCES}

[1] Zukang Shen, Aris Papasakellariou, Juan Montojo, Dirk Gerstenberger and Fangli Xu: "Overview of 3GPP LTEAdvanced Carrier Aggregation for 4G Wireless Communications," IEEE Commun. Mag., Feb. 2012, pp. 112-130.

[2] Guangxiang Yuan ,Xiang Zhang ; Wenbo Wang ; Yang Yang, "Carrier aggregation for LTE advanced mobile communication systems" IEEE Commun. Mag., Feb. 2010, pp. 88-93.

[3] Stephen Wu and Behzad Razavi: "A 900-MHz/1.8-GHz CMOS Receiver for Dual-Band Applications," IEEE J.Solid-State Circuits, Vol. 33, pp. 2178-2185, Dec. 1998.

[4] Kitayabu T., Ikeda Y.: "Implementation of Concurrent Dual-Band Receiver Using IF Undersampling", Radio and Wireless Symposium (RWS), 2011 IEEE.

[5] Kaho, T.; Yamaguchi, Y.; Shiba, H.; Akabane, K.; Uehara, K.; Araki, $\mathrm{K}$, "A simultaneous receiving multi-band mixer with independent gain control", Microwave Conference Proceedings (APMC), 2011 AsiaPacific, 5-8 Dec. 2011, Melbourne, VIC,pp. 383 - 386.

[6] K. Mabrouk, F. Rangel, B. Huyart and G. Neveux: "Architectural solution for second-order intermodulation intercept point improvement in direct down-conversion receivers", IET Microw. Antennas Propag, vol. $4, \mathrm{n}^{\circ}$ 9, pp. 1377-1386, Sept 2010.

[7] C. de la Morena-Álvarez-Palencia, K. Mabrouk, B. Huyart, A. Mbaye, M. Burgos-García: "Direct Baseband I-Q Regeneration Method for Five-Port Receivers Improving DC-offset and Second-Order Intermodulation Distortion Rejection", IEEE Trans. on Microwave Theory \& Techniques, Aug. 2012, vol. 60, n 8, pp. 2634-2643.

[8] K. Mabrouk, B. Huyart and G. Neveux: "3-D Aspect in the Five-Port Technique for Zero-IF Receivers and a New Blind Calibration Method", IEEE Trans. On MTT, june 2008, Vol. 56, pp 1389- 1396. 